\title{
Improving the fatigue life of steel bars by using Nano-coating technology
}

\author{
Ali S.Yasir \\ Mechanical Engineering Department/ University of Kufa, Iraq \\ E-mail:ali_sadiq76@yahoo.com
}

Copyright $\odot 2014$ Ali S.Yasir. This is an open access article distributed under the Creative Commons Attribution License, which permits unrestricted use, distribution, and reproduction in any medium, provided the original work is properly cited.

\begin{abstract}
The fatigue failure is the reason of most mechanical failure for engineering materials. This work tried using the nanocoating technique to coat the steel bars and study the effect of this coating layers on fatigue life of steel. The Zinc Oxide $(\mathrm{ZnO})$ used as it cheap and available in most labs of nanotechnology. The coating process done at different time of coating to get different thickness of coating layers. The result of fatigue tests for coated samples show increased in fatigue life for samples that coated for long time (thick layer of coat), and the increasing if fatigue life may reach to 4 times of its fatigue life for un-coated samples.
\end{abstract}

Keywords: Fatigue, Fatigue Life, Steel Bars, Nano-Coating Technology, ZnO Coat.

\section{Introduction}

Fatigue is the phenomena of failing a component under cyclic loading prior to its ultimate stress. Theories of failure describe the condition for failure. When external stress exceeds a possible value of maximum tensile stress, maximum compressive stress or maximum shear stress the component will fail. In case of fatigue failure, the failure will happen much early to the maximum value of design stress. Fatigue implies changes in properties which can occur in a metallic material due to repeated application of stress and strains, specially to those changes which lead to cracking or failure.[1] The process of fatigue consists of three stages:

- Initial fatigue damage leading to crack initiation.

- $\quad$ Crack propagation to some critical size (size at which the remaining un-cracked cross section of the part becomes too weak to carry the imposed loads).

- $\quad$ Final, sudden fracture of the remaining cross section. [2].

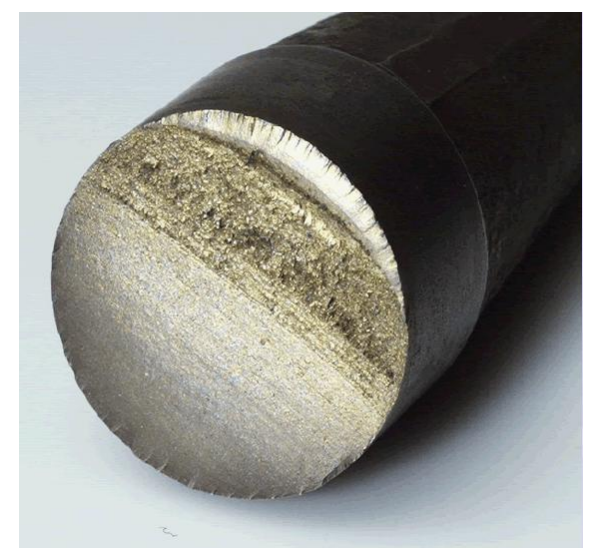

Fig. 1: Shape of Fatigue Failure of Steel Shaft. [3]. 
Masao Kikuch et al. improving the fatigue durability of carburized steels by using the shoot pining with surface structure anomalies (internal oxides precipitated along the grain boundaries and accompanied non-martensitic microstructure near the surface) [4].

$\mathrm{Xu}$ Chen et al. conducted the low-cycle fatigue tests on type 304 stainless steel with loading sequence of axial/torsional, torsional/axial, in phase $190^{\circ}$-out of-phase, and $90^{\circ}$-out of phase /in phase. The results show that cross hardening occurs in axial/torsional loading but not in torsional/axial loading [5].

Jason J.SPICE et al. comparing between three techniques to improve the bending fatigue life, using the gas-carburized, modified Brugger bending fatigue, and actual ring and pinion gears. The gaz-carburized didn't show any improvement in fatigue life for high cycle fatigue but other technique show the improvement in fatigue life. [6].

Ali S.Yasir. Study the effect of cooling rate on the fatigue life of heated steel bar, find that the best fatigue life was for samples that cooled with medium cooling rate (by oil) and the worst fatigue life was for the samples that cooled with high speed cooling (by water and brine). [7].

Nanotechnology is the manipulation or self-assembly of individual atoms, molecules, or molecular clusters into structures to create materials and devices with new or vastly different properties. Nanotechnology can work from the top down (which means reducing the size of the smallest structures to the nanoscale e.g. photonics applications in nanoelectronics and nanoengineering) or the bottom up (which involves manipulating individual atoms and molecules into nanostructures and more closely resembles chemistry biology). [8].

Nanocoating are one of the most important topics within the range of nanotechnology, through the nanoscale engineering of surface and layers.Many synthesis techniques for production of nanostructure coatings have been developed such as sputtering, laser ablation,sol/gel technique ,chemical vapour deposition ,gas condensation, plasma spraying, and electrochemical deposition. [9].

A.Mubarak et al. Study the effect of coating thickness on the properties of (TiN) on tool steel (high speed steel H.S.S) properties by using the physical vapor deposition method. This coating increased hardness of High speed steel. [10]

L.Ipaz et al. improving the mechanical and tribological properties of AISI D3 steel surfaces by coated with (Ti-Al/TiAl-N) multilayer system. The results shows enhancement in hardness, toughness and elastic modulus of steel. [11] Jennifer Hay, Using the Titanium-nitride coating on tool steel to improve the mechanical properties, the result shows improving in surface hardness and modulus of elasticity. [12].

D.Siva Rama Kreshna. Using the $\left(\mathrm{TiO}_{2}\right)$ coating on stainless steel to improving the tribological and corrosion properties of austenitic stainless steels. The results show improving in the hardness and increasing in corrosion resistance and enhancement in wear resistance. [13].

\section{Experimental work}

\subsection{Steel bars (tensile and fatigue test samples) preparation}

The steel bar samples that used in fatigue test before and after coating had the chemical composition as show in table (1).

Table 1: Chemical Composition of Steel Bars.

\begin{tabular}{cccccccccc}
\hline Composition & $\mathrm{C}$ & $\mathrm{Cr}$ & $\mathrm{Ni}$ & $\mathrm{Mn}$ & $\mathrm{Si}$ & $\mathrm{P}$ & $\mathrm{S}$ & $\mathrm{Fe}$ \\
\hline $\mathrm{Wt} \%$ & 0.27 & 0.3 & .3 & 1.6 & 0.55 & 0.04 & 0.04 & Balance \\
\hline
\end{tabular}

The steel bar samples prepared with two shapes:

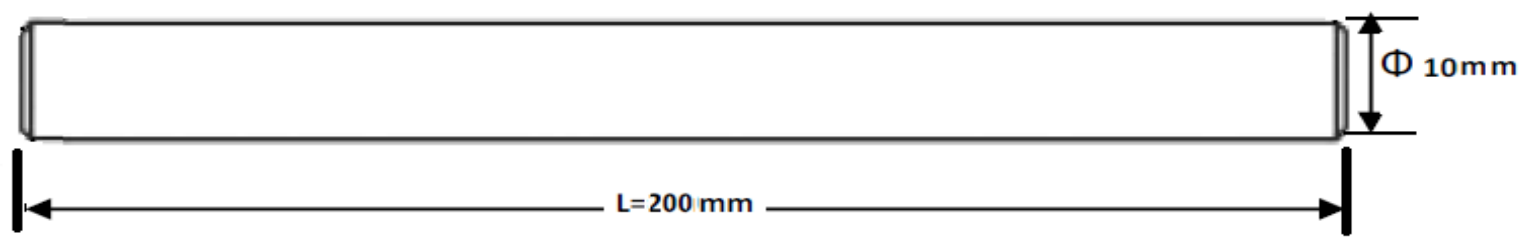

Fig. 2: The Tensile Test Sample

A- Tensile test sample: The tensile test sample figure (2), tested by universal testing machine in figure (3) according to specification (ASTM D683) to get the mechanical properties of steel bar. 


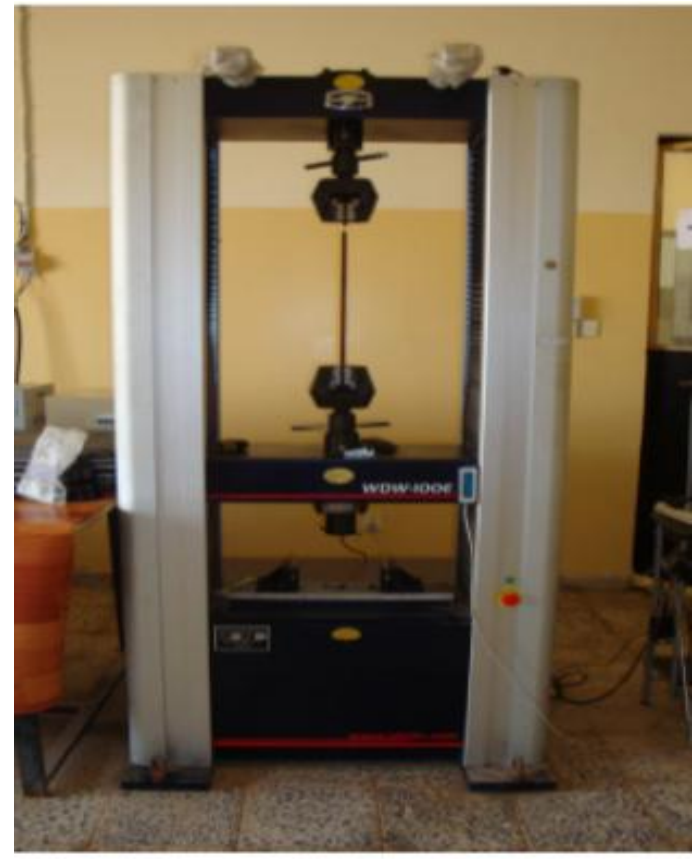

Fig. 3: The Universal Test Machine (UTM)

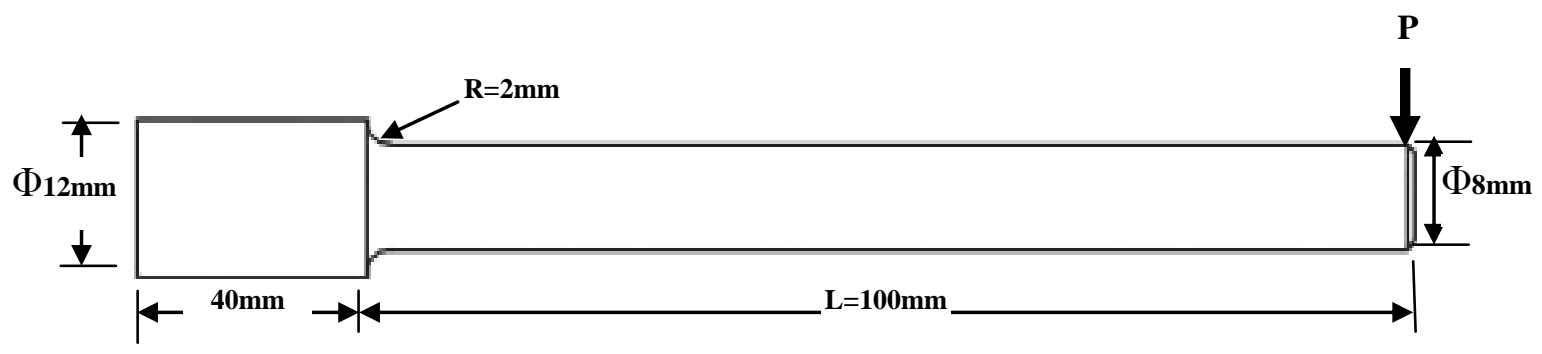

Fig. 4: Fatigue Test Sample

B-Fatigue test samples: The fatigue test samples prepared according to specification (ASTM E467), as shown in (figure.4), and tested by Fatigue test machine that shown in (figure.5), by applying the bending load (150N) at the end of cantilever rotating sample with speed of (2000 r.p.m) of fatigue testing machine .

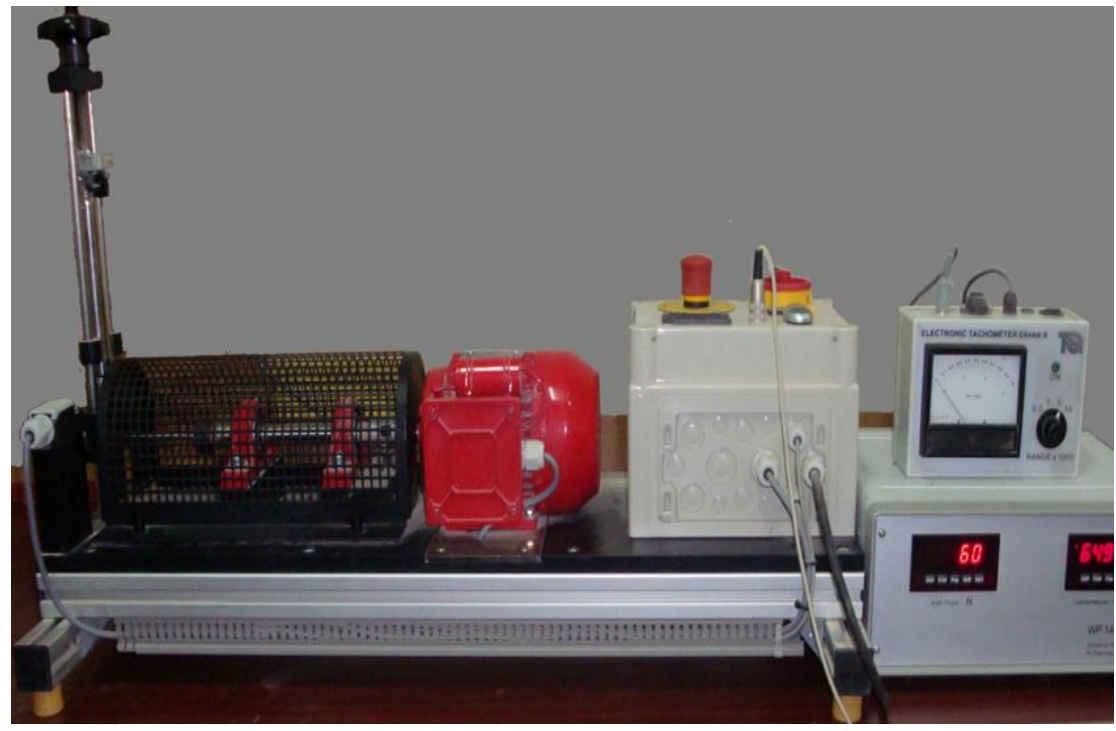

Fig. 5: Fatigue Testing Machine 


\subsection{Nano-coat preparation}

The preparation of nano-coat material by mixing $(4.4 \mathrm{gr})$ of the solution of zinc acetate $\left(\mathrm{Zn}(\mathrm{OAc})_{2} .2 \mathrm{H}_{2} \mathrm{O}\right)$ with $(100$ $\mathrm{mL}$ ) of ethanol in container in centrifuge for one-half hour to ensure the homogeneity. The coating process done inside furnace at temperature of $\left(200 \mathrm{C}^{\circ}\right)$ by using air pump to provide compressed air mixing with nano-coat and applied the stream to steel samples as shown in figure (6). [14].

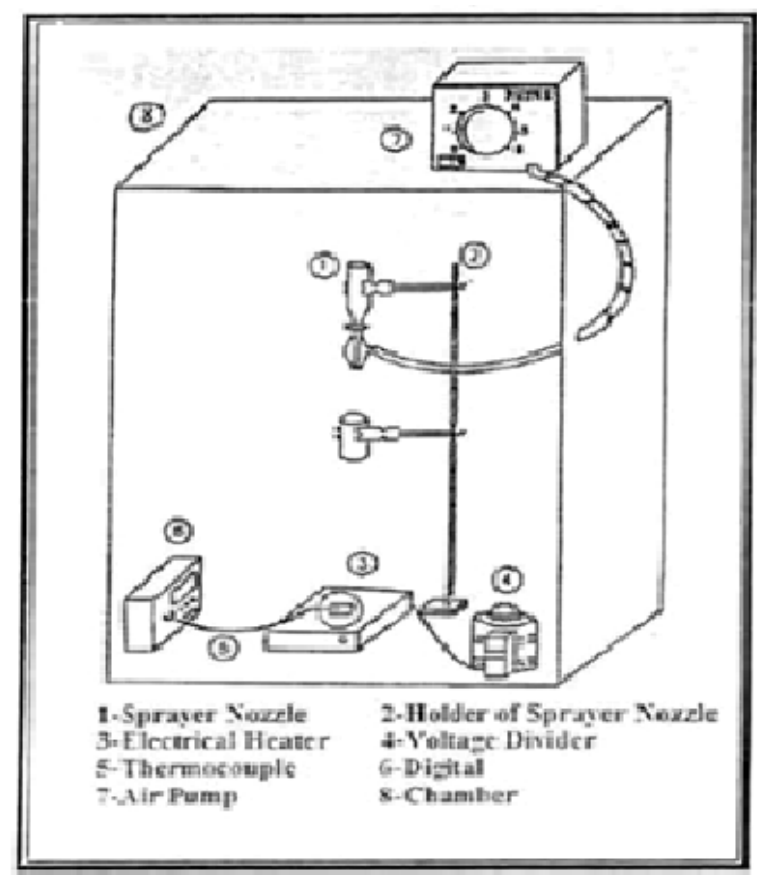

Fig. 6: The Coating Machine for Steel Samples

The glass slide put near the sample that coated to can be determined the thickness of coating layer, the dimension of glass slide are:

Width of glass slide $=24.87 \mathrm{~mm}$.

Length of glass slide $=50.83 \mathrm{~mm}$.

Mass of glass slide before coating $=5.195 \mathrm{gr}$.

Thickness of layer $(\mathrm{t})=\frac{\Delta m}{\mathrm{~A} \rho}$

$\Delta m$--Change in glass slide weight after and before coating (gr).

A-------Area of glass slide $\left(\mathrm{mm}^{2}\right)$.

$\rho$-----Density of coat layer (for $\mathrm{ZnO}$ coating is $5.606 \mathrm{gr} / \mathrm{cm}^{3}$ ).

According to the equation (1) the thickness of coating will be different as different time for coat with average coating rate of $(830 \mathrm{Nm} / \mathrm{sec})$ :

a) Coating time of $(30 \mathrm{sec})$ : That will produce layer of nano-coat of thickness $(2116.6 \mathrm{Nm})$.

b) Coating time of $(60 \mathrm{sec})$ : That will produce layer of nano-coat of thickness $(5291.5 \mathrm{Nm})$.

c) Coating time of $(90 \mathrm{sec})$ : That will produce layer of nano-coat of thickness $(7535.2 \mathrm{Nm})$.

d) Coating time of $(120 \mathrm{sec})$ : That will produce layer of nano-coat of thickness $(10239.7 \mathrm{Nm})$.

Then the samples will put inside furnace at temperature of $\left(450 \mathrm{C}^{\circ}\right)$ to complete formation of zinc oxide $(\mathrm{ZnO})$ nanocoat on the steel samples.

\section{The results and discussion}

3.1. Figure (7) show the results of tensile test for steel sample by universal testing machine, from this figure can find the mechanical properties of steel sample as:

Young modulus $=201 \mathrm{GPa}$

Yield Strength $=575 \mathrm{MPa}$

Ultimate Strength $=675 \mathrm{MPa}$

Maximum strain \% $=0.132$ 


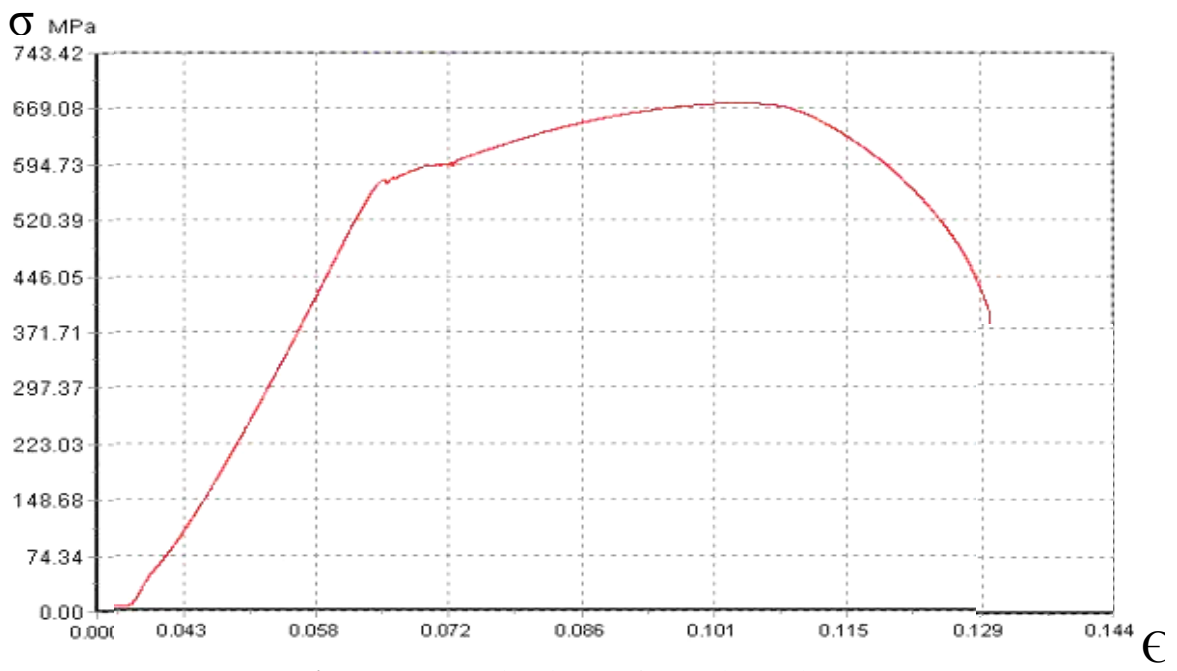

Fig. 7: Stress-Strain Diagram for Steel Sample.

3.2. Figure. (8). show the relation between the time for coating and the thickness of coating layer on the steel sample and can notice the relation is linear with average coating rate $(830$ $\mathrm{nm} / \mathrm{sec})$.

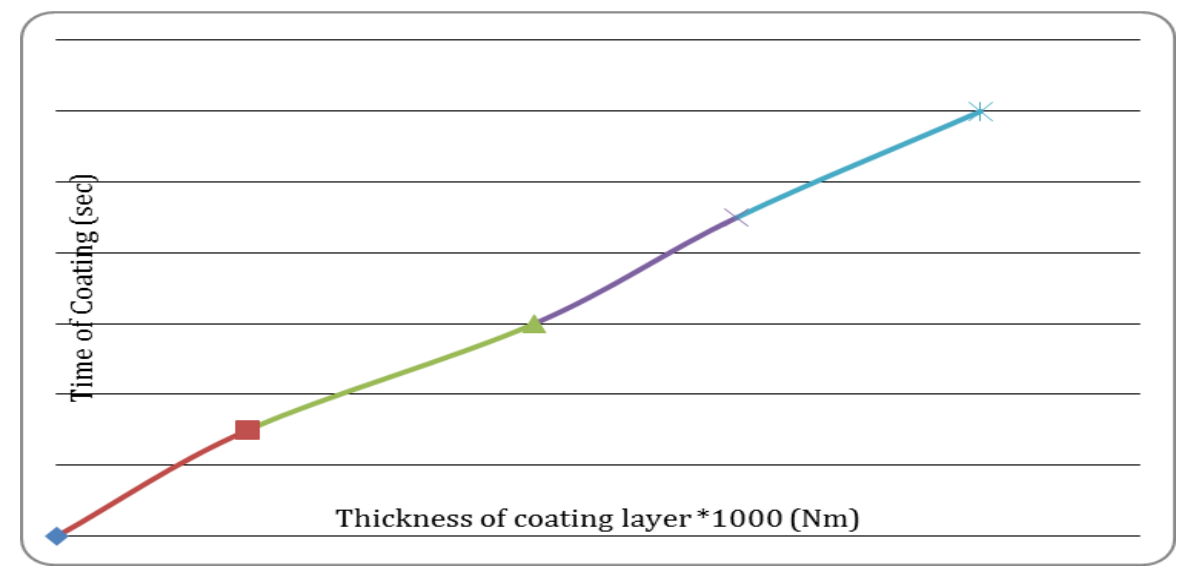

Fig. 8: Relation between the Time of Coating and Thickness of Coating Layer.

3.3. Figure (9) show the relation between the relation between the time of coating and fatigue life (number of cycle) of steel bar, and can notice that the increasing in time of coating will increase the fatigue life of steel bar when sample subjected to fully reversed cyclic load $(r=-1)$.

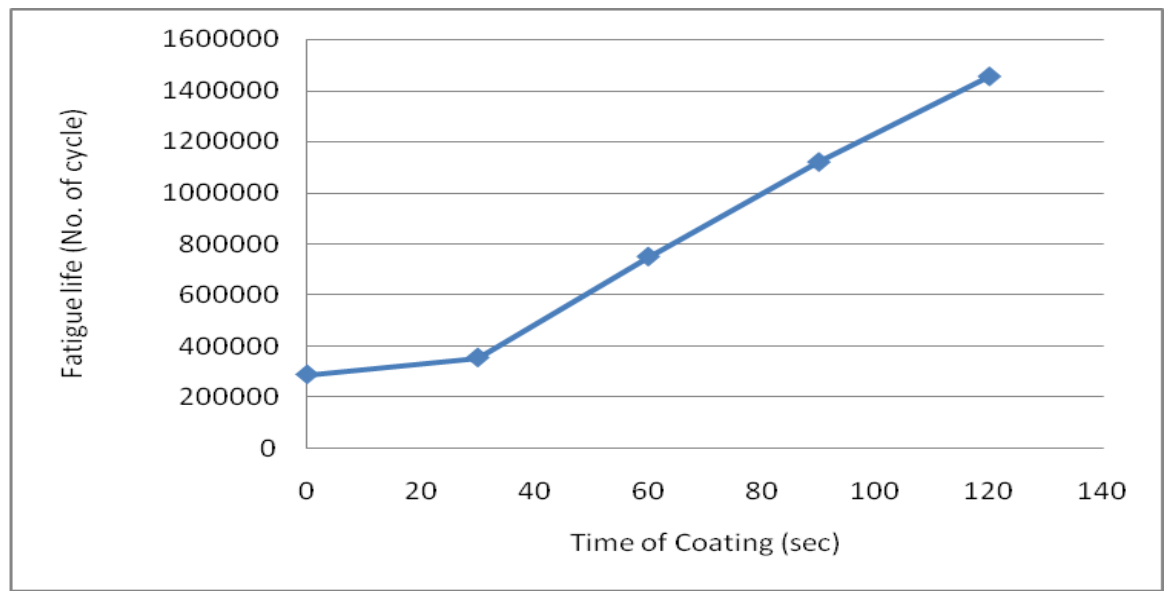

Fig. 9: Relation between Time of Coating and Fatigue Life of Steel Sample. 


\subsection{Figure (10) show the relation between the thickness of coating layer and the fatigue life (no. of cycles) for steel samples.}

From this figure can notice the increasing in fatigue life of steel sample when the thickness of coating layer increased and that happened as the surface of steel will covered by layers of $(\mathrm{ZnO})$ that decrease the initiation of micro cracks and reduced the rate of cracks growing and that increase the fatigue life under fully reversed cyclic load $(\mathrm{R}=-1)$.

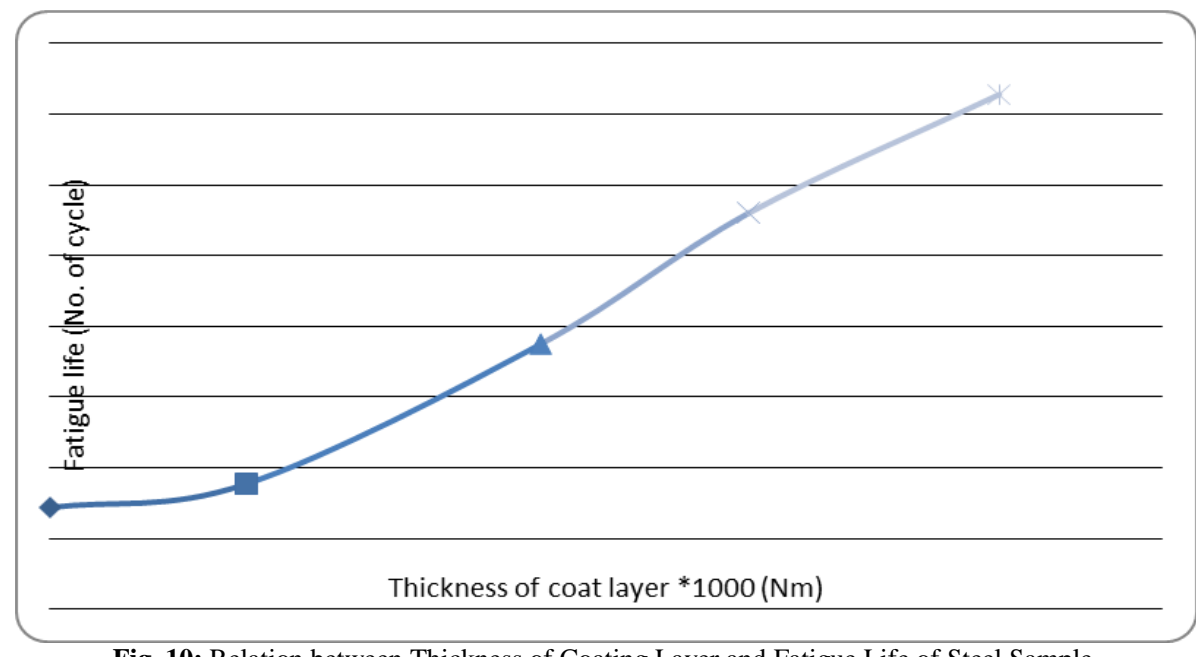

Fig. 10: Relation between Thickness of Coating Layer and Fatigue Life of Steel Sample.

\section{Conclusion}

The result of experimental work of fatigue test show the positive effect of Nano-coating layers of zinc oxide ( $\mathrm{ZnO}$ ) on the fatigue life of rotating steel bars. The improving in fatigue life of rotating steel bar may be reached to about 4-times as nano-coat will reduce the ability to initiation and growing of micro cracks on the steel bar surface that may causes the fatigue failure during rotating under bending load of $(150 \mathrm{~N})$.

\section{Acknowledgements}

I would like to express my thankful and grateful to the staff of nano-technology labs and mechanical engineering labs in university of kufa for support this work.

\section{References}

[1] Shine U. et al, Fatigue Failure of Structural Steel - Analysis Using Fracture Mechanics, World Academy of Science, Engineering and Technology Vol.46., 2008.

[2] Bruce Boardman et al, Fatigue Resistance of Steel, ASM Handbook, Vol.1, pp.673-688, 1990

[3] K. Gopinath, Fatigue Consideration in Design, Machine Design II, 2007.

[4] Masao Kikuchi. Et al., The Improvement of Fatigue Durability of Carburized Steel with Surface Structure Anomalies by Shot Peening, Technical Research Centre. Kumatsu Ltd. Japan. 1986

[5] Xu Chen et al., Fatigue life prediction of type 304 stainless steel under sequential biaxial loading, International Journal of Fatigue, Vol.28, pp.289-299, 2006. http://dx.doi.org/10.1016/j.ijfatigue.2005.05.003.

[6] Jason J Spice. Et al. Optimized Carburized Steel Fatigue Performance as Assessed with Gear and Modified Brugger Fatigue Tests, Society of Automotive Engineers Inc, 2002.

[7] Ali S. Yasir, Study the effect of cooling rate on fatigue strength and fatigue life of heated carbon steel bars, Mechanical Engineering Research; Vol.3, No.2, 2013, pp.1-12, doi:10.5539/ mer.v3n2p. 2013.

[8] Jayanta Kumar Behera, Synthesis and Characterization of ZnO Nano-Particles, Master thesis, National Institute of Technology, India, 2008.

[9] Abdel-Karem R. et al, Nano-coatings, Chapter 6, 2013, http://dx.doi.org/10.5772/55776.

[10] Mubarak. A. et al., Effect of Coating Thickness on The Properties of TiN Coatings Deposited on Tool Steels using Cathodic Arc PVD Technique, Surface Review and Letters, Vol.15,No.4, pp401-410, 2008.

[11] Ipaz. L. et al, Improvement of mechanical and tribological properties in steel surfaces by using Titanium-aluminum/titanium-aluminum nitride multilayered system, Applied Surface Science, ELSEVIER, Vol.258,2012,pp3806-3814,2012

[12] Jennifer Hay, Mechanical Evaluation of Titanium-nitride-coated tool steel. Application Note, 2010.

[13] Siva D. et al., Thermally Oxidized Rutile-TiO2 Coating on Stainless Steel for Tribological Properties and Corrosion Resistance Enhancement, Applied Surface Science, Vol.252, pp1107-1116, 2005. http://dx.doi.org/10.1016/j.apsusc.2005.02.046.

[14] Pilho Huh. et al.(2008), Simple Fabrication of Zinc Oxide Nanostructures., Journal of Materials Chemistry, Vol.18,pp 637-639, DOI: 10.1039/b715334a. http://dx.doi.org/10.1039/b715334a. 